\title{
INTERSECTIONS OF HIGHER-WEIGHT CYCLES OVER QUATERNIONIC MODULAR SURFACES AND MODULAR FORMS OF NEBENTYPUS
}

\author{
BY B. BRENT GORDON ${ }^{1}$
}

In 1976 Hirzebruch and Zagier [6] computed the pairwise intersection multiplicities for a family of algebraic cycles $\left(T_{n}^{c}\right)_{n \in \mathbf{N}}$ in the Hilbert modular surface associated to $\mathbf{Q}(\sqrt{p})$, where $p$ is a prime congruent to $1 \bmod 4$, and showed that the generating function for those intersection multiplicities $\sum_{n=0}^{\infty}\left(T_{m}^{c} \cdot T_{n}^{c}\right) e[n \tau]$ was an elliptic modular form of weight 2 and Nebentypus for $\Gamma_{0}(p)$. Shortly afterwards Zagier [22] observed that if certain weighting factors were attached to the intersection numbers, then the new generating function was again an elliptic modular form of the same level and Nebentypus but now of higher weight. Thus he was led to ask if these weighted intersection numbers could also be realized as the ordinary geometric intersection multiplicities of some algebraic cycles in some appropriate homology theory for the Hilbert modular surface. The purpose of this announcement is to describe an answer to Zagier's question for quaternionic modular surfaces. By combining some ideas of Millson [14] about higher-weight cycles in torus bundles over locally symmetric spaces with the notion of an algebraically defined subspace in the cohomology of a Kuga fiber variety (cf. [4]), we are able to associate to each Hirzebruch-Zagier cycle $T_{n}$ in a quaternionic modular surface $X$ an algebraic cycle $\tau_{n}$ in a family of abelian varieties $A$ over $X$ such that: (a) The pairwise intersection multiplicities $\left(\tau_{m} \cdot \tau_{n}\right)$ of these cycles have precisely the same form as Zagier's weighted intersection numbers (Theorem 1), and (b) the generating function for intersection numbers $\sum_{n=0}^{\infty}\left(\tau_{m} \cdot \tau_{n}\right) e[n \tau]$ is an elliptic modular form of higher weight and Nebentypus for an appropriate $\Gamma_{0}(N) \subset \mathrm{SL}_{2}(\mathbf{Z})$ (Theorem 2).

Tong [20] also looked at Zagier's question. In 1979 his response was to associate to each Hirzebruch-Zagier cycle a current in the cohomology of the Hilbert modular surface with coefficients in a complex vector bundle and show, using the theory of [19], that the intersection multiplicities of these currents coincided with Zagier's weighted intersection numbers. Now it is no coincidence that the cohomology classes represented by the cycles $\tau_{n}$ live in a subspace $H^{\bullet}(\mathcal{M})$ of $H^{\bullet}(\mathcal{A}, \mathbf{Q})$ which is isomorphic to the vector-bundle-valued cohomology that Tong worked with (cf. Lemma 1 below), and that under such an isomorphism the cycles $\tau_{n}$ correspond to his currents. The point is that the whole picture can be realized algebraically inside of the variety $A$ (Proposition $1)$, so that one could start a priori with the "motive" $H^{\bullet}(\mathcal{M})$. Moreover, the

Received by the editors August 25, 1985.

1980 Mathematics Subject Classification (1985 Revision). Primary 10D21, $10 \mathrm{D} 12$.

${ }^{1}$ Research partially supported by the SFB "Theoretische Mathematik" and the MaxPlanck-Institut für Mathematik, Bonn, and the University of Maryland. 
Eichler-Shimura isomorphism identifies this $H^{\bullet}(\mathcal{M})$ with a space of cusp forms of higher weight for an arithmetic subgroup of $\mathrm{SL}_{2}(\mathbf{R}) \times \mathrm{SL}_{2}(\mathbf{R})$ (cf. [13 and 11], Chapter II). Thus the present work could also be viewed as an algebraicgeometric formulation of the kind of lifting of automorphic forms which arises from restricting the Weil representation to the groups of a dual reductive pair; see [15 and 9], for example.

I would like to thank Zagier for suggesting this problem to me as well as for his continuing interest and encouragement. I also profited from some conversations with Kudla.

1. Let $V$ be a 4-dimensional vector space over $\mathbf{Q}$ equipped with an anisotropic quadratic form $q$ of signature $(2,2)$, and let $L$ be a lattice in $V$ on which $q$ is integer-valued. Assume that the discriminant $D$ of $L$ is not a square. Then the even Clifford algebra $C^{+}(V)$ is a totally indefinite division quaternion algebra over the real quadratic field $\mathbf{Q}(\sqrt{D})$; its reduced discriminant is generated by the product of those rational primes at which $q$ is anisotropic; and it contains the even Clifford algebra $C^{+}(L)$ as an order. Moreover, for 4-dimensional $V$ the spin group $G:=\operatorname{Spin}(V)$ is the group of norm 1 units in $C^{+}(V)$. Thus $G$ is simple over $\mathbf{Q}$ while $G_{\mathbf{R}} \simeq \mathrm{SL}_{2}(\mathbf{R}) \times \mathrm{SL}_{2}(\mathbf{R})$. Let $\psi: G \rightarrow \mathrm{SO}(V)$ denote the natural representation of $G$ on $V$, and let $\lambda$ denote the left regular representation of $G$ on $C^{+}(V)$. Next choose an integer $k \geq 0$ and let $M$ be a free $\mathbf{Z}$-module of rank $k$. We include the case $k=0$ for completeness and take $M=\{0\}$ when $k=0$. Then $\Lambda:=C^{+}(L) \otimes \mathbf{z} M$ is a lattice in $W:=C^{+}(V) \otimes \mathbf{z} M$, and the representation $\sigma:=\lambda \otimes 1$ of $G$ on $W$ is equivalent over $\mathbf{Q}$ to $k$ copies of the spin representation of $G$.

Now let $\Gamma$ be any torsion-free normal subgroup of finite index in $C^{+}(L) \cap G$. (Such groups exist by [1].) Then $\psi(\Gamma)$ preserves $L$ and $\sigma(\Gamma)$ preserves $\Lambda$. Denote the symmetric domain associated to $G$ by $\mathfrak{D}$, i.e. $\mathfrak{D} \simeq G_{\mathbf{R}} / K$ for a maximal compact subgroup $K$ of $G_{\mathbf{R}}$. Then it is well known (cf. [7]) that $\mathcal{X}:=\Gamma \backslash \mathfrak{D}$ can be embedded as a complex projective algebraic surface. What's more, it follows from the work of Kuga $[\mathbf{1 1}]$ and Satake $[\mathbf{1 7}, \mathbf{1 8}]$ that the torus bundle $A:=\Gamma \backslash\left(\mathfrak{D} \times\left(W_{\mathbf{R}} / \Lambda\right)\right)$ over $\mathcal{X}$ can also be embedded as a complex projective algebraic variety with the structure of a family of polarized abelian varieties parameterized by $\chi$.

LEMMA 1. Let $(\rho, E)$ be the absolutely irreducible representation of $G$ defined over $\mathbf{Q}$ of highest weight $(2 k, 2 k)$. Then there exists a unique subspace $H^{4 k+2}(\mathcal{M}) \subset H^{4 k+2}(A)$ such that $H^{4 k+2}(\mathcal{M}) \simeq H^{2}(\Gamma, E)$.

Here we take cohomology with coefficients in $\mathbf{Q}$ unless otherwise indicated. With the observation that $\rho$ occurs with multiplicity one in $\bigwedge^{4 k} \sigma$ (cf. [11, Lemma IV-2-1]), this lemma follows, for example, from [12, (1.3.3)]. Then the following proposition identifies $H^{4 k+2}(\mathcal{M})$, defined by the lemma, as being an algebraically defined subspace of $H^{4 k+2}(\mathcal{A})$, that is, essentially the Betti realization of a motive (cf. $[2, \S 0]$ ).

PROPOSITION 1. There exists an algebraic cycle $P \subset A \times A$ of (complex) dimension $4 k+2$ such that the projection from $H^{4 k+2}(A)$ to $H^{4 k+2}(\mathcal{M})$ can be obtained by lifting a class from $H^{4 k+2}(\mathcal{A})$ to $H^{4 k+2}(\mathcal{A} \times \mathcal{A})$ via the first 
projection from $A \times A$ to $A$, then taking the cup product with the class that $P$ represents, $[P] \in H^{8 k+4}(A \times A)$, and then taking the image in $H^{4 k+2}(A)$ under the Gysin homomorphism associated to the second projection from $A \times A$ to A. In particular, algebraic classes in $H^{4 k+2}(A)$ project to algebraic classes in $H^{4 k+2}(\mathcal{M})$, and a class in $H^{4 k+2}(\mathcal{M})$ is algebraic if and only if it is algebraic as a class in $H^{4 k+2}(\AA)$.

This proposition is a special case of $[\mathbf{4}$, Theorem 1$]$.

2. Let $($,$) denote the symmetric bilinear form on V$ defined by $(u, v):=$ $q(u+v)-q(u)-q(v)$. For nonzero $v \in L$, let $V_{v}$ denote the orthogonal complement of $\mathbf{Q} v$ in $V$, and let $L_{v}:=L \cap V_{v}$. The following lemma is actually true for any quadratic lattice over a principal ideal domain so long as $v$ is not isotropic.

LEMMA 2. Let $n_{L}(v):=$ g.c.d. $\{(v, w) \mid w \in L\}$. Then the discriminant $D_{v}$ of $L_{v}$ is related to the discriminant $D$ of $L$ by $D_{v}=n_{L}(v)^{-2}(v, v) D$.

Now let $L^{+}:=\{v \in L \mid q(v)>0\}$. Then for $v \in L^{+}$we can construct an algebraic family of abelian varieties $A_{v}$ associated to $V_{v}$ and $L_{v}$ just as before: The even Clifford algebra $C^{+}\left(V_{v}\right)$ is an indefinite division quaternion algebra over $\mathbf{Q}$ contained in $C^{+}(V)$; it contains $C^{+}\left(L_{v}\right)$ as an order; $G_{v}$ can be defined as $G \cap G^{+}\left(V_{v}\right)$, or as $\operatorname{Spin}\left(V_{v}\right)$, or as the stabilizer of $\mathbf{Q} v$ in $G$; let $W_{v}:=C^{+}\left(V_{v}\right) \otimes \mathbf{z} M$ and $\Lambda_{v}:=C^{+}\left(L_{v}\right) \otimes \mathbf{z} M$; let $\Gamma_{v}:=\Gamma \cap G_{v}$; and let $\mathfrak{D}_{v}$ be the hermitian symmetric domain associated to $G_{v}$. Then as before $A_{v}:=\Gamma_{v} \backslash\left(\mathfrak{D}_{v} \times\left(W_{v \mathbf{R}} / \Lambda_{v}\right)\right)$ can be embedded as a complex projective variety with the structure of a family of polarized abelian varieties parameterized by $X_{v}:=\Gamma_{v} \backslash \mathfrak{D}_{v}$. Moreover, the natural inclusions of $\mathfrak{D}_{v}$ and $\mathfrak{D}_{v} \times W_{v \mathbf{R}}$ in $\mathfrak{D}$ and $\mathfrak{D} \times W_{\mathbf{R}}$, respectively, induce holomorphic immersions $i_{v}:=X_{v} \rightarrow \chi$ and $h_{v}:=A_{v} \rightarrow A$ compatible with the fiber structure of $A_{v}$ and $A$.

DEFINITION. (a) For $v \in L^{+}$, let

$$
\tau_{v}:=n_{L}(v)^{2 k} p_{2^{*}}\left(P \cdot\left(h_{v}\left(A_{v}\right) \times A\right)\right),
$$

where $P$ is an algebraic cycle in $A \times A$ with the properties described in Proposition 1 , and $(\cdot)$ denotes the intersection product (in the sense of rational homology) in $A \times A$, and $p_{2 *}$ is the map on cycles induced by the second projection from $A \times A$ to $A$, and $n_{L}(v)$ is defined as in Lemma 2. Then $T_{v}$ is an algebraic cycle of higher weight which represents a well-defined class in $H^{4 k+2}(A)$. In fact, $\left[\tau_{v}\right]=P\left(n_{L}(v)^{2 k}\left[h_{v}\left(A_{v}\right)\right]\right)$, where $P$ denotes the projection from $H^{4 k+2}(\mathcal{A})$ to $H^{4 k+2}(\mathcal{M})$.

(b) For $m \in \mathbf{Z}_{+}$, let

$$
\tau_{m}:=\sum_{v \in \Gamma \backslash L(m)} \tau_{v},
$$

where $L(m):=\{v \in L \mid q(v)=m\}$. We call $\tau_{m}$ an arithmetic cycle of higher weight, as its definition depends on the arithmetic of $L$.

(c) Let $\tau_{0}$ be defined formally by

$$
\left[\tau_{0}\right]:= \begin{cases}2^{-1} c_{1} & \text { if } k=0 \\ 0 & \text { if } k>0\end{cases}
$$


where $c_{1}$ is the first Chern class on $\chi$.

The arithmetic cycles of higher weight are the analogs of the HirzebruchZagier cycles in the present case. Of course if $k=0$ then $\tau_{m}$ is not of higher weight at all, rather it coincides with the Hirzebruch-Zagier cycle $T_{m}$ in $\chi$. On the other hand, when $k>0$ then $\tau_{m}$ lives over $T_{m}$ in the sense that

$$
T_{m}=\phi_{*}\left(\sum_{v \in \Gamma \backslash L(m)} h_{v}\left(\mathcal{A}_{v}\right)\right)=\sum_{v \in \Gamma \backslash L(m)} i_{v}\left(\mathcal{X}_{v}\right)
$$

where $\phi_{*}$ is the map on cycles associated to the natural projection $\phi: A \rightarrow X$.

3. Let $\left(\tau_{m} \cdot \tau_{n}\right)$ denote the intersection multiplicity of $\tau_{m}$ with $\tau_{n}$ in the sense of rational homology.

THEOREM 1.

$$
\left(\tau_{m} \cdot \tau_{n}\right)=\sum_{\substack{(v, w) \in \Gamma \backslash(L(m) \times L(n)) \\ D(v, w)<0}} P_{2 k}(v, w)+\sum_{\substack{(v, w) \in \Gamma \backslash(L(m) \times L(n)) \\ D(v, w)=0}} E\left(X_{v}\right) P_{2 k}(v, w)
$$

where $D(v, w):=(v, w)^{2}-4 q(v) q(w)$,

$$
P_{2 k}(v, w):=\sum_{j=0}^{k}(-1)^{j\left(\begin{array}{c}
2 k-j \\
j
\end{array}\right)} q(v)^{j} q(w)^{j}(v, w)^{2 k-2 j}
$$

and $E\left(X_{v}\right)$ is the Euler volume of $X_{v}$.

Some remarks concerning this theorem might be in order. First of all, notice that $D(v, w)$ is the discriminant of $q$ restricted to $\mathbf{Z} v+\mathbf{Z} w$. So when $v$ or $w$ is in $L^{+}$, then $D(v, w)<0$ if and only if this binary quadratic form is positive definite. On the other hand, for nonzero $v$ and $w$ then $D(v, w)=0$ if and only if $\mathbf{Q} v=\mathbf{Q} w$, in which case $h_{v}\left(A_{v}\right)=h_{w}\left(A_{w}\right)$ and $\tau_{v}$ is a multiple of $\tau_{w}$. Thus the second term in the expression for $\left(\tau_{m} \cdot \tau_{n}\right)$ comes from the self-intersection multiplicities of the common components of $\tau_{m}$ and $\tau_{n}$-this is why the Euler number appears. In any case the second term vanishes unless $m n$ is a square.

Secondly, note that the polynomial $P_{2 k}(v, w)$ is homogeneous of degree $2 k$ in $v$ and $w$ separately. In fact $P_{2 k}(v, w)=q(v)^{k} q(w)^{k} C_{2 k}^{1}\left(\left(v^{\prime}, w^{\prime}\right)\right)$, where $C_{2 k}^{1}$ is the Gegenbauer, or ultraspherical, polynomial (cf. [21, Chapter IX], or your favorite text on orthogonal polynomials) and $u^{\prime}:=(u, u)^{-1 / 2} u \in V_{\mathbf{R}}$ is the unit vector in the $u$-direction for any $u \in L^{+}$.

As for the proof of Theorem 1 , if $k=0$ then the methods of [8] suffice. On the other hand, when $k>0$ we can write down the harmonic differential form $\eta_{m}$ on $A$ whic represents $\left[\tau_{m}\right]$, and then integrate $\eta_{m}$ over $\tau_{n}$. Unfortunately this is a somewhat lengthy business, as it must begin with choosing coordinates on $\mathcal{A}$. Eventually, however, the computations on which the proof depends are just like Zagier's [22, Theorem 6 and 23, Theorem 2].

4. THEOREM 2. The Fourier series

$$
F_{m}(\tau):=\sum_{n=0}^{\infty}\left(\tau_{m} \cdot \tau_{n}\right) e[n \tau]
$$


is an elliptic modular form of weight $2 k+2$ on $\Gamma_{0}(N)$ with character $\chi$, where $N$ and $\chi$ are the level and character, respectively, of $L$ (in the sense of $[5, \S 4])$. If $k>0$ then $F_{m}(\tau)$ is a cusp form.

The proof of Theorem 2 from Theorem 1 is quite amusing. The starting point is the following proposition, which can be proved by combinatorial arguments in the spirit of [8] or can be deduced from [10]. Let $Z:=\left(\begin{array}{ll}\tau & z \\ z & \tau^{\prime}\end{array}\right)$ denote an element of the Siegel upper half-plane of genus 2 .

Proposition 2. The Fourier series

$$
\begin{aligned}
\Phi(Z) & :=2^{-1} E(\mathcal{X})+\sum_{\substack{(v, w) \in \Gamma \backslash\left(L^{+} \times L^{+}\right) \\
D(v, w)<0}} e\left[q(v) \tau+(v, w) z+q(w) \tau^{\prime}\right] \\
+ & E\left(\chi_{v}\right) e\left[q(v) \tau+(v, w) z+q(w) \tau^{\prime}\right] \\
& \sum_{\substack{(v, w) \in \Gamma \backslash\left(L^{+} \times L^{+}\right) \\
D(v, w)=0}}
\end{aligned}
$$

is a Siegel modular form of genus 2 , weight 2 and character $\chi$ for $\Gamma_{0}^{(2)}(N) \subset$ $\mathrm{Sp}_{4}(\mathbf{Z})$.

In fact $\Phi(Z)$ is the theta function for $\Gamma$-inequivalent representations of positive semidefinite binary quadratic forms in $L$.

So if we expand $\Phi(Z)$ in a Fourier series as a function of $\tau^{\prime}$, then it follows immediately from the proposition that each Fourier coefficient $\phi_{m}(\tau, z)$, for $m \in \mathbf{N}$, is a Jacobi form of weight 2 and index $m$, in the terminology of $[\mathbf{3}]$, for $\Gamma_{0}(N) \subset \mathrm{SL}_{2}(\mathrm{Z})$ with character $\chi$ (cf. [3, Theorem 6.1, or 16]). And now suddenly Theorem 2 follows as a special case of [3, Theorem 3.1]! For it is readily checked that $F_{m}(\tau)$ is the $2 k$ th "development coefficient" of $\phi_{m}(\tau, z)$, meaning that it is derived from the $2 k$ th Taylor coefficient of $\phi_{m}(\tau, z)$ at $z=0$ in such a way that it becomes an elliptic modular form of weight $2 k+2$ also for $\Gamma_{0}(N)$ with character $\chi$.

REMARK. It should be noted that more notations but no new ideas are needed to generalize the methods and results described in this note to the case where $V$ is a 4-dimensional vector space over a totally real number field $F$ and $q$ is an anisotropic quadratic form on $V$ which has signature $(2,2)$ at some of the real places of $F$ and $(4,0)$ at the rest.

\section{REFERENCES}

1. A. Borel, Compact Clifford-Klein forms of symmetric spaces, Topology 2 (1963), 111-132.

2. P. Deligne, Valeurs de fonctions $L$ et périods d'intégrales, Automorphic forms, representations and L-functions, Proc. Sympos. Pure Math., vol. 33, Amer. Math. Soc., Providence, R. I., 1979, pp. 313-346.

3. M. Eichler and D. Zagier, The theory of Jacobi forms, Progress in Math. 55, Birkhäuser, Boston, Basel, Stuttgart, 1985.

4. B. B. Gordon, Algebraically defined subspaces in the cohomology of a Kuga fiber variety, preprint.

5. E. Hecke, Analytische arithmetik der positiven quadratischen formen, Mathematische Werke, 789-918, Vanderhoeck and Ruprecht, Göttingen, 1970. 
6. F. Hirzebruch and D. Zagier, Intersection numbers of curves on Hilbert modular surfaces and modular forms of Nebentypus, Invent. Math. 36 (1976), 57-113.

7. K. Kodaira, On Kähler varieties of restricted type, Ann. of Math. (2) 60 (1954), 28-48.

8. S. S. Kudla, Intersection numbers for quotients of the complex 2-ball and Hilbert modular forms, Invent. Math. 47 (1978), 189-208.

9. S. Kudla and J. Millson, Geodesic cycles and the Weil representation I; quotients of hyperbolic space and Siegel modular forms, Compositio Math. 45 (1982), 207-271.

10. , The theta correspondence and harmonic forms. I and II, preprint.

11. M. Kuga, Fibre varieties over a symmetric space whose fibres are abelian varieties, vols. I and II, Univ. of Chicago, 1964.

12. __ Algebraic cycles in gtfabv, J. Fac. Sci. Univ. Tokyo 29 (1982), 13-29.

13. Y. Matsushima and G. Shimura, On the cohomology groups attached to certain vector valued differential forms on the product of the upper half planes, Ann. of Math. (2) 78 (1963), 417-449.

14. J. Millson, Higher weight cycles, J. Fac. Sci. Univ. of Tokyo 28 (1981), 465-479.

15. T. Oda, On modular forms associated with indefinite quadratic forms of signature (2,n-2), Math. Ann. 231 (1977), 97-144.

16. I. I. Pyatetskii-Shapiro, Automornhic forms and the geometry of classical domains, Gordon and Breach, New York, 1969.

17. I. Satake, Clifford algebras and families of abelian varieties, Nagoya Math. J. 27 (1966), 435-446. Corrections ibid. 31 (1968), 295-296.

18. _ _ Algebraic structures of symmetric domains, Iwanami Shoten, Tokyo and Princeton Univ. Press, Princeton, 1980.

19. D. Toledo and Y. L. L. Tong, Duality and intersection theory in complex manifolds. I, Math. Ann. 237 (1978), 41-77.

20. Y. L. L. Tong, Weighted intersection numbers on Hilbert modular surfaces, Compositio Math. 38 (1979), 299-310.

21. N. J. Vilenkin, Special functions and the theory of group representations, Transl. Math. Monographs, vol. 22, Amer. Math. Soc., Providence, R. I., 1968.

22. D. Zagier, Modular forms whose Fourier coefficients involve zeta-functions of quadratic fields, Modular functions of one variable VI, Bonn, Lecture Notes in Math., vol. 627, Springer-Verlag, New York, 1976.

23. __ The Eichler-Selberg trace formula, appendix to S. Lang, Introduction to modular forms, Springer-Verlag, New York, 1976.

\section{Department of Mathematics, Purdue University, West Lafayette, IN- DIANA 47907}

Current address: Department of Mathematics, University of Oklahoma, Norman, Oklahoma 73019 